\title{
Exact k-Wise Intersection Theorems
}

\section{Journal Article}

\section{Author(s):}

Szabó, Tibor; Vu, V.H.

Publication date:

2005-06

Permanent link:

https://doi.org/10.3929/ethz-b-000032661

Rights / license:

In Copyright - Non-Commercial Use Permitted

Originally published in:

Graphs and combinatorics 21(2), https://doi.org/10.1007/s00373-005-0609-0 


\title{
Exact $k$-Wise Intersection Theorems
}

\author{
Tibor Szabó ${ }^{1, *}$ and V.H. Vu ${ }^{2, *}$ \\ ${ }^{1}$ Department of Computer Science, ETH, Zürich, 8092, Switzerland. e-mail: \\ szabo@inf.ethz.ch \\ ${ }^{2}$ Department of Mathematics, UCSD, La Jolla, CA92093-0112, USA. \\ e-mail:vanvu@ucsd.edu
}

\begin{abstract}
A typical problem in extremal combinatorics is the following. Given a large number $n$ and a set $L$, find the maximum cardinality of a family of subsets of a ground set of $n$ elements such that the intersection of any two subsets has cardinality in $L$.

We investigate the generalization of this problem, where intersections of more than 2 subsets are considered. In particular, we prove that when $k-1$ is a power of 2 , the size of the extremal $k$-wise oddtown family is $(k-1)\left(n-2 \log _{2}(k-1)\right)$. Tight bounds are also found in several other basic cases.
\end{abstract}

\section{Introduction}

In this paper we study families $\mathscr{F}$ of subsets of an $n$-element ground set $[n]=\{1,2, \ldots, n\}$, for which the size of the intersection of any $k$ members of $\mathscr{F}$ is in a prescribed set of integers. On the most general level our problem can be formulated as follows.

Given a positive integer $n$, a set $L$ of integers, and an integer $k=k(n)>1$ find the maximum cardinality of a family of subsets of $[n]$ such that the intersection of any $k$ of these subsets has a cardinality contained in $L$.

For $k=2$, these types of questions have been attacked successfully using linear algebraic methods. The excellent monograph [2] by Babai and Frankl contains a wide variety of results and applications. In the following we list some of the most basic theorems. Sets of even (odd) cardinality are called even (odd). A condition on the $k$-wise intersection is always about $k$ pairwise distinct members of the family.

\footnotetext{
* Research supported in part by NSF grant DMS 99-70270 and by the joint Berlin/Zurich graduate program Combinatorics, Geometry, Computation, financed by the German Science Foundation (DFG) and ETH Zürich

${ }^{\dagger}$ Research supported in part by NSF grant DMS-0200357, by an NSF CAREER award and by an Alfred P. Sloan fellowship. webpage: http://www.math.ucsd.edu/vanvu/
} 
Eventown Theorem (Berlekamp [5], Graver [11]). Let $\mathscr{F} \subseteq 2^{[n]}$ be a family such that the intersection of any two subsets in $\mathscr{F}$ is even. Then $|\mathscr{F}| \leq 2^{\lfloor n / 2\rfloor}+\delta_{n}$, where $\delta_{n}=1$ if $n$ is odd and 0 otherwise.

Oddtown Theorem (Berlekamp [5]). Let $\mathscr{F} \subseteq 2^{[n]}$ be a family of odd subsets, such that the intersection of any two subsets in $\mathscr{F}$ is even. Then $|\mathscr{F}| \leq n$.

Nonuniform Fischer Inequality (Majumdar [14]). Let $\mathscr{F} \subseteq 2^{[n]}$ be a family such that the intersection of any two subsets in $\mathscr{F}$ has the same nonzero cardinality. Then $|\mathscr{F}| \leq n$.

Nonuniform Ray-Chaudhuri-Wilson Theorem (Frankl and Wilson [6]). Given a subset $L$ of s nonnegative integers, let $\mathscr{F} \subseteq 2^{[n]}$ be a family such that the intersection of any two members of $\mathscr{F}$ has a cardinality contained in L. Then $|\mathscr{F}| \leq \sum_{i=0}^{s}\left(\begin{array}{c}n \\ i\end{array}\right)$.

It is important to note that all the above bounds are best possible.

The generalization of these theorems from pairwise to $k$-wise intersections seems completely natural, still the adaptation of the arguments poses some nontrivial challenge. The reason for this difficulty lies exactly where the beauty of the proofs for $k=2$ is. Arguments about pairwise intersections usually depend on the basic fact that the size of the intersection of two sets is equal to the inner product of the two corresponding characteristic vectors, hence the machinery of linear algebra can be invoked. The intrinsic difficulty of the $k$-wise case compared to the pairwise is the lack of algebraic concepts describing the intersection of $k$ sets when $k>2$. In this paper we try to circumvent this obstacle with additional combinatorial ideas while still making some use of linear algebra.

The general problem of investigating $k$-wise intersection restrictions on families of sets for $k \geq 3$ was posed in [15] by V. T. Sós. Füredi [8] has established a combinatorial connection between the pairwise and $k$-wise case for $t$-uniform systems. He showed that for any fixed $L$, the order of magnitude of the size of the extremal system for the pairwise and $k$-wise problem is the same. His constants are very large, but depend only on $k$ and $t$. The $k$-wise variant of specific intersection problems were studied recently by the second author [16,17], Grolmusz [12], and Grolmusz and Sudakov [13].

An important feature of the linear algebra method is that the obtained results are often tight. There are, usually quite simple, matching constructions complementing the algebraic upper bounds; not just up to a constant factor or asymptotically, but precisely. Sharp results, just because of their scarcity, are of particular interest in Extremal Combinatorics. In this paper we put the emphasis on obtaining precise results for the $k$-wise version of some of the classical pairwise intersection theorems, like the Oddtown Theorem, or the Nonuniform Fischer Inequality.

\section{1 k-wise Results}

A precise bound for the $k$-wise version of the Eventown Theorem was given by the second author in an earlier paper [16]. Here we quote this result for the sake of completeness. 
Theorem 1.1. There is a positive constant $c$ such that the following holds. If $n \geq c \log _{2} k$ and $\mathscr{F} \subseteq 2^{[n]}$ is a family of maximum size such that the intersection of every $k$ subsets in $\mathscr{F}$ is even, then $|\mathscr{F}|=2^{\lfloor n / 2\rfloor}+\delta_{k, n}$, where $\delta_{k, n}=k-1$ if $n$ is odd and 0 otherwise.

We call a family $\mathscr{F} \subseteq 2^{[n]} k$-wise oddtown, if every member of $\mathscr{F}$ is odd and the intersection of any $k$ members is even. The following is conjectured to be the optimal family if $n$ is large enough compared to $k$.

Construction A. [16] Let us consider $b=\left\lceil\log _{2}(k-1)\right\rceil$ disjoint 2-element subsets of $[n]$, say $X_{i}=\{2 i-1,2 i\}$ for $i=1, \ldots, b$. By taking the unions of some of these pairs, create $k-1$ sets $Y_{1}, \ldots, Y_{k-1}$; say let $Y_{i}:=X_{j_{1}} \cup \cdots \cup X_{j_{i r}}$, where $j_{1}, \ldots, j_{i_{r}}$ are the nonzero coordinates in the binary expansion of $i-1$. Define

$$
\mathscr{F}=\left\{Y_{i} \cup\{x\}: i=1, \ldots, k-1 ; x=2 b+1, \ldots, n\right\} .
$$

Then $|\mathscr{F}|=(k-1)\left(n-2\left\lceil\log _{2}(k-1)\right\rceil\right)$ and $\mathscr{F}$ is $k$-wise oddtown. Indeed, no $k$-wise intersection contains anything from $\{2 b+1, \ldots, n\}$, while points from $[2 b]$ come in pairs.

In the following theorem we confirm that for an infinite sequence of $k$ Construction $\mathrm{A}$ is, indeed, extremal. We also obtain an almost tight bound for every $k$.

Theorem 1.2. Let $k=k(n) \geq 3$ be an integer and $\mathscr{F} \subseteq 2^{[n]}$ be a $k$-wise oddtown family of maximum size. If $k=2^{s}+1$ for some integer $s$, then for every $n \geq 3 \frac{k-1}{k-2} \log _{2}(k-1)$

$$
|\mathscr{F}|=(k-1)\left(n-2 \log _{2}(k-1)\right) \text {. }
$$

Moreover, for every $k$ and $n \geq 3(k-1)+2\left\lceil\log _{2}(k-1)\right\rceil-2$,

$$
(k-1)\left(n-2\left\lceil\log _{2}(k-1)\right\rceil\right) \leq|\mathscr{F}| \leq(k-1)\left(n-\left\lfloor\log _{2}(k-1)\right\rfloor-\left\lceil\log _{2}(k-1)\right\rceil\right) .
$$

We consider Theorem 1.2 the main contribution of our paper. Previously the asymptotics of the size of the maximum family was established [16] for fixed $k$, but exact results were not known for any $k>2$.

We also prove the following precise $k$-wise version of the Nonuniform RayChaudhuri-Wilson Theorem for $|L|=1$.

Theorem 1.3. Let $\mathscr{F} \subseteq 2^{[n]}$ be a largest family such that the intersections of any $k=k(n)$ subsets in $\mathscr{F}$ has the same cardinality. If $k-1>2^{n-1}$, then $|\mathscr{F}|=2^{n}$. Otherwise let $s$ be the largest integer such that $\sum_{i=0}^{s-1}\left(\begin{array}{c}n-1 \\ i\end{array}\right) \leq k-1$. Then

$$
|\mathscr{F}|=\sum_{i=0}^{s}\left(\begin{array}{l}
n \\
i
\end{array}\right)+\left\lfloor\left(k-1-\sum_{i=0}^{s-1}\left(\begin{array}{c}
n-1 \\
i
\end{array}\right)\right) \frac{n}{s+1}\right\rfloor
$$


In particular for $n \geq k$, we have

$$
|\mathscr{F}|=\left\lfloor\frac{k}{2} n\right\rfloor+1 .
$$

This theorem improves on the earlier bound of $(k-1)(n+1)$ of Grolmusz and Sudakov [13].

For pairwise intersections, the Nonuniform Ray-Chaudhuri-Wilson Theorem is sharp only when $L=\{0\}$. In case $L \neq\{0\}$, the Nonuniform Fischer Inequality improves the upper bound $n+1$ to $n$. A similar phenomenon occurs here as well: Theorem 1.3 is only sharp if all $k$-wise intersections are empty. The following statement is a $k$-wise variant of the Nonuniform Fischer Inequality.

Theorem 1.4. Assume that $\mathscr{F} \subseteq 2^{X}$ is a maximum size family such that the intersection of any $k=k(n)$ members of $\mathscr{F}$ has the same nonzero cardinality. Then

$$
|\mathscr{F}|=\left\{\begin{array}{cc}
f(k, n-1) & \text { if } k-1 \leq 2^{n-2} \\
k-1 & \text { otherwise }
\end{array}\right.
$$

where $f(k, n)$ is the quantity on the right hand side of (1).

Further questions one may want to consider are the $k$-wise variants of the famous modular intersection theorems of Frankl and Wilson. The uniform version could be considered a far-reaching generalization of the Oddtown Theorem. Here we discuss the nonuniform case.

Nonuniform Frankl-Wilson Theorem [6]. Let $p$ be a prime and $L$ be the set of $s$ residues modulo $p$. If $\mathscr{F} \subseteq 2^{[n]}$ is a family such that for every $A \in \mathscr{F},|A| \notin L(\bmod p)$ and for every $A, B \in \mathscr{F},|A \cap B| \in L(\bmod p)$, then

$$
|\mathscr{F}| \leq \sum_{i=0}^{s}\left(\begin{array}{l}
n \\
i
\end{array}\right) .
$$

In a recent paper [13], Grolmusz and Sudakov proved the upper bound $(k-1) \sum_{i=0}^{s}\left(\begin{array}{l}n \\ i\end{array}\right)$ for the $k$-wise version of the above statement. Using an idea from the proof of Theorem 1.1, we can slightly improve their bound.

Proposition 1.5. Let $p$ be a prime and $L$ be a set of $s$ residues modulo $p$. Suppose $\mathscr{F} \subseteq 2^{[n]}$ is a family, such that for every $A \in \mathscr{F},|A| \notin L(\bmod p)$ and for every $A_{1}, \ldots, A_{k} \in \mathscr{F},\left|\cap_{I=1}^{k} A_{i}\right| \in L(\bmod p)$. If $\sum_{i=0}^{s}\left(\begin{array}{c}n \\ i\end{array}\right) \geq\left\lfloor\log _{p}(k-1)\right\rfloor$, then

$$
|\mathscr{F}| \leq(k-1)\left(\sum_{i=0}^{s}\left(\begin{array}{l}
n \\
i
\end{array}\right)-\left\lfloor\log _{p}(k-1)\right\rfloor+1\right) .
$$

The rest of the paper is organized as follows. In the next section, we describe some useful ideas for the proof of Theorem 1.2 and prove Proposition 1.5. The proof of Theorem 1.2 follows in Section 3. In Section 4 we prove Theorems 1.3 and 1.4 , while the last section contains several concluding remarks and open questions. 
Notation. The degree $\operatorname{deg}_{\mathscr{F}}(x)$ of a point $x$ in a family $\mathscr{F}$ is the number of members of $\mathscr{F}$ containing $x$.

In Sections 2 and 3 we use the language of linear algebra. For each subset $A$ of $[n]$, the characteristic vector of $A$ is the binary vector $v_{A}$ of length $n$, whose $i^{t h}$ coordinate is 1 if $i$ belongs to $A$ and 0 otherwise. The vector space generated by the characteristic vectors of the elements of $\mathscr{F}$ over the two-element field $G F(2)$ is denoted by $V(\mathscr{F})$.

\section{Warm-up}

In this section, we describe some ideas which will be used in the proof of Theorem 1.2. First we discuss a weaker upper bound, proved by the second author as a lemma to the proof of Theorem 1.1 ([Lemma 6,16]; see also [17, Lemma 3.2.4] for a more general statement). Then, we modify the proof of this weaker bound to prove Proposition 1.5.

\subsection{A Weaker Bound for the Oddtown Problem}

Lemma 2.1. [16] If $\mathscr{F} \subseteq 2^{[n]}$ is a k-wise oddtown family and $\operatorname{dim} V(\mathscr{F})=$ $d>\left\lfloor\log _{2}(k-1)\right\rfloor$ then,

$$
|\mathscr{F}| \leq(k-1)\left(d-\left\lfloor\log _{2}(k-1)\right\rfloor\right)
$$

Proof. We use induction on $d$. The base case $d=\left\lfloor\log _{2}(k-1)\right\rfloor+1$ is immediate, since in any finite dimensional vector space over $G F(2)$ at most half of the vectors have odd weight, thus

$$
|\mathscr{F}| \leq \frac{1}{2}|V(\mathscr{F})|=2^{d-1} \leq(k-1)
$$

Assume now that $d>\left\lfloor\log _{2}(k-1)\right\rfloor+1$. Let $t$ be the largest integer such that there are $t$ members $A_{1}, \ldots, A_{t}$ of $\mathscr{F}$ having an odd intersection $A=\cap_{i=1}^{t} A_{i}$. Clearly $t \leq k-1$. Then for every $i=1, \ldots, t$,

$$
v_{A_{i}} v_{A}=\left|A_{i} \cap A\right|=|A| \equiv 1(\bmod 2) .
$$

Due to the maximality of $t$, for any set $B \in \mathscr{F}^{\prime}=\mathscr{F} \backslash\left\{A_{1}, \ldots, A_{t}\right\}$

$$
v_{B} v_{A}=|B \cap A| \equiv 0(\bmod 2) .
$$

It thus follows that the vectors $v_{A_{i}}$ are not contained in the space $V\left(\mathscr{F}^{\prime}\right)$ generated by the family $\mathscr{F}^{\prime}=\mathscr{F} \backslash\left\{A_{1}, \ldots, A_{t}\right\}$. Thus the dimension of $V\left(\mathscr{F}^{\prime}\right) \leq d-1$ and this, together with the induction hypothesis, yields 
$\left.|\mathscr{F}|=t+\left|\mathscr{F}^{\prime}\right| \leq(k-1)+(k-1)\left((d-1)-\left\lfloor\log _{2}(k-1)\right\rfloor\right)\right)=(k-1)\left(d-\left\lfloor\log _{2}(k-1)\right\rfloor\right)$,

completing the proof.

Corollary 2.2. [16] Let $\mathscr{F}$ be a $k$-wise oddtown family on $n>\left\lfloor\log _{2}(k-1)\right\rfloor$ points. Then $|\mathscr{F}| \leq(k-1)\left(n-\left\lfloor\log _{2}(k-1)\right\rfloor\right)$.

Proof. It is clear that $\operatorname{dim} V(\mathscr{F}) \leq n$.

The consideration of the dimension of $V(\mathscr{F})$ turns out to be a very useful concept and will play a crucial role in the proof of Theorem 1.2. But first let us use the previous idea to show Proposition 1.5.

\subsection{Proof of Proposition 1.5}

Consider a family $\mathscr{F}$ as in Proposition 1.5. We mimic the method of Alon, Babai and Suzuki [1] for the proof of the Frankl-Wilson Theorem and combine it with the ideas in the previous argument. Let $W$ be the vector space of all polynomials generated by the set $\left\{\prod_{i \in S} x_{i}|S \subset\{1,2, \ldots, n\}| S \mid, \leq s\right\}$ over the field $G F(p)$. It is easy to see that the generating set forms a basis as well, thus the dimension of $W$ is $\sum_{i=0}^{s}\left(\begin{array}{c}n \\ i\end{array}\right)$.

To each set $A \subset X$, assign a polynomial $\tilde{f}_{A}(x)=\prod_{l \in L}\left(x \cdot v_{A}-l\right)$, where $x$ denotes a vector in $G F^{n}(p)$. Let $f_{A}(x)$ be the multilinear polynomial we obtain from $\tilde{f}_{A}(x)$ by replacing all powers $x_{i}^{q}$ by the first power $x_{i}$. Clearly $f_{A}(y)=\tilde{f}_{A}(y)$ for any 0 1 -vector $y$. Let $d$ denote the dimension of the subspace (of $W$ ) spanned by the polynomials $f_{B}, B \in \mathscr{F}$. We shall prove, by induction on $d$, that if $d \geq\left\lfloor\log _{p}(k-1)\right\rfloor$, then

$$
|\mathscr{F}| \leq(k-1)\left(d-\left\lfloor\log _{p}(k-1)\right\rfloor+1\right)
$$

As $d \leq \operatorname{dim}(W)=\sum_{i=0}^{s}\left(\begin{array}{c}n \\ i\end{array}\right)$, our claim follows.

Let $d=\left\lfloor\log _{p}(k-1)\right\rfloor$. A subspace of $W$ with dimension $d$ has $p^{d}$ vectors, so it follows that

$$
|\mathscr{F}| \leq p^{d} \leq(k-1)
$$

concluding the proof of the base case.

Now assume $d>\left\lfloor\log _{p}(k-1)\right\rfloor$. Similar to the previous proof, let $t$ be the largest integer such that there are $t$ members of $\mathscr{F}$ whose intersection has cardinality not in $L$ modulo $p$. Let these members be $A_{1}, \ldots, A_{t}$ and let $A=\cap_{i=1}^{t} A_{i}$. It follows that for every $i=1, \ldots, t$,

$$
f_{A_{i}}\left(v_{A}\right)=\tilde{f}_{A_{i}}\left(v_{A}\right)=\prod_{l \in L}\left(\left|A_{i} \cap A\right|-l\right)=\prod_{l \in L}(|A|-l) \not \equiv 0(\bmod p) .
$$

On the other hand, due to the maximality of $t$, for any member $B \in \mathscr{F}^{\prime}=\mathscr{F} \backslash\left\{A_{1}, \ldots, A_{t}\right\},\left|B \cap\left(\cap_{i=1}^{t} A_{i}\right)\right|=|B \cap A| \in L$. Therefore, 


$$
f_{B}\left(v_{A}\right)=\tilde{f}_{B}\left(v_{A}\right)=\prod_{l \in L}(|B \cap A|-l) \equiv 0(\bmod p) .
$$

Hence for every $i, 1 \leq i \leq t, f_{A_{i}} \in W$ is linearly independent from the subspace $V^{\prime}$ spanned by the $f_{B}, B \in \mathscr{F}^{\prime}$. Thus $\operatorname{dim} V^{\prime} \leq d-1$ and we can conclude as in the previous proof.

\section{3. $k$-wise Oddtown}

In order to prove Theorem 1.2, we need to consider a more general scenario, where $\mathscr{F}$ is allowed to contain sets with high multiplicity. This extension will provide room for us to consider a more general induction hypothesis. To this end we need to introduce a few definitions. A collection $\mathscr{F}$ of sets is called an l-multisystem if each set occurs in $\mathscr{F}$ with multiplicity at most $l$. A 1 -multi-system is called a family. We extend the definition of the $k$-wise oddtown property to multisystems. A multi-system $\mathscr{F}$ is said to have the $k$-wise oddtown property if

- $|A| \equiv 1(\bmod 2)$ for every $A \in \mathscr{F}$ and

- $\left|A_{1} \cap \ldots \cap A_{k}\right| \equiv 0(\bmod 2)$ for every sub-multi-system $\left\{A_{1}, \ldots, A_{k}\right\}$ of $\mathscr{F}$.

That is, in the intersection property, $A_{i}$ and $A_{j}$ might be equal as sets, but they are distinct members of the multi-system. Note that in a $k$-wise oddtown multisystem there cannot be a set with multiplicity larger than $k-1$.

Proof of Theorem 1.2. First we shall prove that for an extremal $k$-wise oddtown system $\mathscr{F}, \operatorname{dim} V(\mathscr{F})$ should be at most $n-\left\lceil\log _{2}(k-1)\right\rceil$ and then apply Lemma 2.1 .

The following definition plays a crucial role in our argument.

Definition. Let $\mathscr{F}$ be a multi-system of sets. A set $M$ is called a basis set of $\mathscr{F}$, if

(i) $M$ is nonempty

(ii) $|M|$ is even

(iii) $M=A_{1} \cap \ldots \cap A_{k-1}$ for some sub-multi-system $\left\{A_{1}, \ldots, A_{k-1}\right\}$ of $\mathscr{F}$.

Observe, that the characteristic vector $w$ of a basis set $M=A_{1} \cap \ldots \cap A_{k-1}$ is orthogonal to $V(\mathscr{F})$. Indeed, for any $B \in \mathscr{F}, B \neq A_{i}, i=1, \ldots, k-1, w \cdot v_{B}=0$ since $\mathscr{F}$ is a $k$-wise oddtown system, while $w \cdot v_{A_{i}}=0$ because of $(i i)$. Thus the vector space $W(\mathscr{F})$, generated by the characteristic vectors of the basis sets of $\mathscr{F}$, is contained in the orthogonal complement $V(\mathscr{F})^{\perp}$ of $V(\mathscr{F})$. It is well-known that for any subspace $V, \operatorname{dim} V+\operatorname{dim} V^{\perp}=n$. Therefore $d \leq n-b$, where $b=\operatorname{dim} W(\mathscr{F})$.

In the following theorem we prove that the maximum size of a $k$-wise oddtown family with $\operatorname{dim} W(\mathscr{F})<\left\lceil\log _{2}(k-1)\right\rceil$ is asymptotically much less than $(k-1)\left(n-2\left\lceil\log _{2}(k-1)\right\rceil\right)$, the lower bound given by Construction A. 
Theorem 3.1 Let $k \geq 3, l, n \geq 1$ be integers and $\mathscr{F} \subseteq 2^{[n]}$ be a $k$-wise oddtown l-multi-system. Let $b \leq k-2$ be the dimension of the vector space $W(\mathscr{F})$ generated by the characteristic vectors of the basis sets of $\mathscr{F}$. Then

$$
|\mathscr{F}| \leq \max _{i=0}^{b}\left\{\frac{2^{i+1} l+k-1}{3}(n-2 i)\right\} .
$$

Proof of Theorem 3.1. We proceed by induction on $b$. To check the base case $b=0$, assume that $\mathscr{F}$ is a family with no basis sets, that is all $(k-1)$-wise intersections are either odd or empty. We use induction on $n$. For $n=1$, the maximum size system contains the singleton $\min \{l, k-1\} \leq(2 l+k-1) / 3$-times.

Suppose now that $n>1$.

Case 1. Every point is contained in at most $k-1$ sets. Then

$$
\sum_{A \in \mathscr{F}}|A|=\sum_{A \in \mathscr{F}} \sum_{p \in A} 1=\sum_{p} \sum_{p \in A \in \mathscr{F}} 1 \leq n(k-1) .
$$

The number of sets in $\mathscr{F}$ is maximized when (almost) all degrees are $k-1$ and the sizes of the sets are as small as possible. That is when $\mathscr{F}$ contains all the 1-element subsets with multiplicity $\min \{l, k-1\}$ and a 3-uniform (almost-) regular hypergraph with maximum degree $(k-1-\min \{l, k-1\}){ }^{1}$ Then $|\mathscr{F}| \leq \min \{l, k-1\} n+(k-1-\min \{l, k-1\}) n / 3 \leq(2 l+k-1) n / 3$.

Case 2. There is a point $x$ with degree at least $k$.

Then there are some $A_{1}, \ldots, A_{k}$, such that their intersection contains $x$. Since this intersection is even, it must contain another element $y \neq x$. But $A_{1} \cap \ldots \cap A_{k-1}$ is then not empty, thus it is odd (remember that $b=0$ ), so $N=A_{1} \cap \ldots \cap A_{k-2}$ contains at least three elements.

Consider those members $E$ of $\mathscr{F}\left(E \neq A_{i}, i=1, \ldots, k-2\right)$ for which $E \cap N$ is not empty, thus odd. There are at most $|N|$ of such $E$ 's. (For any two $E_{1}, E_{2}$, $N \cap E_{1} \cap E_{2}$ is even. This implies that an odd intersection $N \cap E$ determines $E$ uniquely. Then one can use the pairwise Oddtown Theorem within $N$ for the sets $N \cap E$, when this intersection is odd.)

All other members of $\mathscr{F}$ are disjoint from $N$, thus they form a system on $n-|N|$ points. So by induction

$$
\begin{aligned}
|\mathscr{F}| & \leq \frac{(2 l+k-1)(n-|N|)}{3}+|N|+k-2 \\
& =\frac{2 l+k-1}{3} n-\frac{|N|}{3}(2 l+k-4)+k-2 \leq \frac{2 l+k-1}{3} n,
\end{aligned}
$$

since $|N| \geq 3$ and $l \geq 1$.

\footnotetext{
${ }^{1}$ Remark: In case $k \leq\left(\begin{array}{c}n-1 \\ 2\end{array}\right)+2$, Baranyai's Theorem ensures that this actually can be achieved, such that, depending on the remainder of $n$ modulo 3 , only 0,1 or 2 points have degree smaller than the maximum.
} 
This completes the proof of the base case $b=0$. Now let us assume that $b>0$. Let us choose a minimal basis set $M=A_{1} \cap \ldots \cap A_{k-1}$.

If $|M|=2$, then by the $k$-wise oddtown property each element of $\mathscr{F}$ either contains or is disjoint from $M$. Then the multi-system $\mathscr{F} \backslash M=\{E \backslash M: E \in \mathscr{F}\}$ is a $k$-wise oddtown $2 l$-multi-system on $n-2$ points. The dimension of $W(\mathscr{F} \backslash M)$ is $b-1$. (More precisely, $W(\mathscr{F})$ is equal to the direct product $W(\mathscr{F} \backslash M) \times\left\langle v_{M}\right\rangle$. If there is a basis set $B$ of $\mathscr{F} \backslash M$ which is not a basis set of $\mathscr{F}$, then $B \cup M$ is a basis set of $\mathscr{F}$, thus the characteristic vector of $B$ is in $W(\mathscr{F})$. On the other hand if $B$ is basis set of $\mathscr{F}$ then $B \backslash M$ is a basis set of $\mathscr{F} \backslash M$.) Thus, by the induction hypothesis,

$$
\begin{aligned}
|\mathscr{F}| & =|\mathscr{F} \backslash M| \leq \max _{i=0}^{b-1}\left\{\frac{2^{i+1} \cdot 2 l+k-1}{3}((n-2)-2 i)\right\} \\
& =\max _{j=1}^{b}\left\{\frac{2^{j+1} \cdot l+k-1}{3}(n-2 j)\right\} .
\end{aligned}
$$

Suppose now that $|M| \geq 4$.

Case 1. There is an index $\mathrm{j}$ such that $\cap_{i \neq j} A_{i} \backslash A_{j}=\emptyset$.

Then the $(k-2)$-wise intersection $N=\cap_{i \neq j} A_{i}$ would be equal to $M$ and would not contain any other basis set because of the minimality of $M$. Thus, again, every member of $\mathscr{F}$ either contains or is disjoint from $M$, implying that $\mathscr{F} \backslash M$ is a $k$ wise $2 l$-multi-system. Similarly, by the induction hypothesis,

$$
\begin{aligned}
|\mathscr{F}| & =|\mathscr{F} \backslash M| \leq \max _{i=0}^{b-1}\left\{\frac{2^{i+1} \cdot 2 l+k-1}{3}((n-|M|)-2 i)\right\} \\
& \leq \max _{j=1}^{b}\left\{\frac{2^{j+1} \cdot l+k-1}{3}(n-2 j)\right\} .
\end{aligned}
$$

Case 2. For every index $j=1, \ldots, k-1$, the sets $C_{j}=\cap_{i \neq j} A_{i} \backslash A_{j}$ are not empty.

Let us choose some $k-2$ sets from $\left\{A_{1}, \ldots A_{k-1}\right\}$ such that their intersection does not contain any other basis set, but $M$. This is possible because of a simple counting argument. We say that a basis set $B \neq M$ ruins an index $j$ if $B \subseteq \cap_{i \neq j} A_{i}$. In case index $j$ is ruined, let us fix an arbitrary basis set $B_{j}$ ruining $j$. Then the minimality of $M$ implies $B_{j} \cap C_{j} \neq \emptyset$. Since the nonempty $C_{j}$ s are pairwise disjoint by definition, the set of characteristic vectors $\left\{v_{B_{j}}: j\right.$ is ruined $\}$ is linearly independent. Hence $b$, the dimension of the space of the characteristic vectors of basis sets, cannot be less than (actually it is equal to) the number of indices ruined. Since $b \leq k-2$, we have an index, say $k-1$, which is not ruined by any basis set. Then $N=\cap_{i=1}^{k-2} A_{i}$ does not contain any other basis set, but $M$.

In particular, the intersection of any member of $\mathscr{F} \backslash\left\{A_{1}, \ldots, A_{k-2}\right\}$ with $N$ is either odd, or $M$, or empty. Let 


$$
\begin{aligned}
& \mathscr{F}_{N}=\left\{A_{1}, \ldots, A_{k-2}\right\} \\
& \mathscr{F}_{0}=\left\{E \in \mathscr{F} \backslash \mathscr{F}_{N}: E \cap N=\emptyset\right\}, \\
& \mathscr{F}_{1}=\left\{E \in \mathscr{F} \backslash \mathscr{F}_{N}:|E \cap N| \equiv 1(\bmod 2)\right\}, \\
& \mathscr{F}_{2}=\left\{E \in \mathscr{F} \backslash \mathscr{F}_{N}: 0 \neq|E \cap N| \equiv 0(\bmod 2)\right\}=\left\{E \in \mathscr{F} \backslash \mathscr{F}_{N}: E \cap N=M\right\} .
\end{aligned}
$$

The family $\mathscr{F}_{1}^{\prime}=\left\{E \cap N: E \in \mathscr{F}_{1}\right\}$ is a pairwise oddtown family on $|N|$ points, so $\left|\mathscr{F}_{1}\right|=\left|\mathscr{F}_{1}^{\prime}\right| \leq|N|$.

Any element $E$ of $\widetilde{\mathscr{F}}_{0} \cup \mathscr{F}_{2}$ is either disjoint from $N$ or intersects it in $M$. Then $\left(\mathscr{F}_{0} \cup \mathscr{F}_{2}\right) \backslash N=\left\{E \backslash N: E \in \mathscr{F}_{0} \cup \mathscr{F}_{2}\right\}$ is a $k$-wise oddtown 2 -multisystem on $n-|N|$ points. Let the dimension of $W\left(\left(\mathscr{F}_{0} \cup \mathscr{F}_{2}\right) \backslash N\right)$ be $b^{\prime}$. If $B$ is a basis set of $\left(\mathscr{F}_{0} \cup \mathscr{F}_{2}\right) \backslash N$ then either $B$ itself or $B \cup M$ is a basis set of $\mathscr{F}$. In any case the characteristic vector $v_{B}$ is in $W(\mathscr{F})$. Hence $W\left(\left(\mathscr{F}_{0} \cup \mathscr{F}_{2}\right) \backslash M\right) \subseteq W(\mathscr{F})$. Thus $b^{\prime} \leq b-1$, since $v_{M} \in W(\mathscr{F}) \backslash W\left(\left(\mathscr{F}_{0} \cup \mathscr{F}_{2}\right) \backslash M\right)$. By the induction hypothesis,

$$
\begin{aligned}
|\mathscr{F}| & =\left|\mathscr{F}_{N}\right|+\left|\mathscr{F}_{1}\right|+\left|\mathscr{F}_{0} \cup \mathscr{F}_{2}\right| \leq k-2+|N|+\max _{i=0}^{b^{\prime}}\left\{\frac{2^{i+1} \cdot 2 l+k-1}{3}(n-|N|-2 i)\right\} \\
& =\max _{i=0}^{b^{\prime}}\left\{\frac{2^{i+2} l+k-1}{3}(n-2(i+1))-|N| \frac{2^{i+2} l+k-4}{3}+\frac{2^{i+3} l+2 k-2+3 k-6}{3}\right\} \\
& \leq \max _{i=0}^{b^{\prime}}\left\{\frac{2^{i+2} l+k-1}{3}(n-2(i+1))-5 \frac{2^{i+2} l+k-4}{3}+\frac{2^{i+3} l+5 k-8}{3}\right\}= \\
& =\max _{i=0}^{b^{\prime}}\left\{\frac{2^{i+2} l+k-1}{3}(n-2(i+1))-\frac{3 \cdot 2^{i+2} l-12}{3}\right\} \leq \\
& \leq \max _{i=0}^{b^{\prime}}\left\{\frac{2^{i+2} l+k-1}{3}(n-2(i+1))\right\} \leq \max _{j=1}^{b}\left\{\frac{2^{j+1} l+k-1}{3}(n-2 j)\right\}
\end{aligned}
$$

Here we used that $|N| \geq 5$, since $|M| \geq 4$ and $C_{j} \neq \emptyset$.

The proof of Theorem 1.2 is now immediate by applying Theorem 3.1 with $l=1$. Suppose first that $k-1=2^{s}$ for some positive integer $s$. The size of the family from Construction A is larger than the upper bound in (2) for sufficiently large $n$. Indeed, $(k-1)(n-2 s)$ is larger than $\frac{2^{b+1}+k-1}{3}(n-2 b)$ for every $b<s$ if $n \geq 3 \log _{2}(k-1) \cdot \frac{k-1}{k-2}$. Hence for an extremal system $\mathscr{F}$, the dimension of $W(\mathscr{F})$ cannot be less than $\log _{2}(k-1)$, which implies that $\operatorname{dim} V(\mathscr{F}) \leq n-\log _{2}(k-1)$. Then the first part of Theorem 1.2 is a consequence of Lemma 2.1.

Assume now that $2^{s}+1 \leq k-1<2^{s+1}$. Our argument is similar to the above, except the range of validity is somewhat smaller. For any $n \geq 3(k-1)+2\left\lceil\log _{2}(k-1)\right\rceil-2$, the size of the family from Construction $\mathrm{A}$ is greater than the dominating term $\frac{2^{s+1}+k-1}{3}(n-2 s)$ in the upper bound (2). So, again, $\operatorname{dim} V(\mathscr{F}) \leq n-\left\lceil\log _{2}(k-1)\right\rceil$ and the second part of Theorem 1.2 follows from Lemma 2.1 . 


\section{4. $k$-wise Nonuniform Fischer-Inequality}

In this section we prove Theorems 1.3 and 1.4 .

It is convenient to introduce the following definition. We say that $\mathscr{F}$ is a $k$-wise $l$-Fischer family if the intersection of any $k$ members of $\mathscr{F}$ contains exactly $l$ elements. Let $m_{l}^{k}(n)$ be the largest possible size of a $k$-wise $l$-Fischer setsystem. First we determine $m_{0}^{k}(n)$ exactly for every $n$ and $k$.

\section{Lemma 4.1.}

$$
m_{0}^{k}(n)= \begin{cases}\sum_{i=0}^{s}\left(\begin{array}{c}
n \\
i
\end{array}\right)+\left\lfloor\left(k-1-\sum_{i=0}^{s-1}\left(\begin{array}{c}
n-1 \\
i
\end{array}\right)\right) \frac{n}{s+1}\right\rfloor & \text { if } k-1 \leq 2^{n-1} \\
2^{n} & \text { if } k-1>2^{n-1}\end{cases}
$$

where $s$ is the largest integer such that $\sum_{i=0}^{s-1}\left(\begin{array}{c}n-1 \\ i\end{array}\right) \leq k-1$ (provided s exists, i.e. $\left.k-1 \leq 2^{n-1}\right)$.

Proof. For a family $\mathscr{H}$ let us denote by $D(\mathscr{H})$ the sum of the degrees:

$$
D(\mathscr{H}):=\sum_{v \in V} \operatorname{deg}_{\mathscr{H}}(v)=\sum_{A \in \mathscr{H}}|A|
$$

The heart of the proof is the following trivial observation.

Observation. A family is $k$-wise 0 -Fischer if and only if each point has degree at most $k-1$.

For $k-1 \geq 2^{n-1}$ the lemma follows easily from our observation since the degree of any point in $2^{[n]}$ is $2^{n-1} \leq k-1$. Suppose now that $\sum_{i=0}^{s-1}\left(\begin{array}{c}n-1 \\ i\end{array}\right) \leq k-1<\sum_{i=0}^{s}\left(\begin{array}{c}n-1 \\ i\end{array}\right)$. First we construct an appropriate family $\mathscr{F}$ of the desired size. Let us denote by $\left(\begin{array}{c}{[n]} \\ i\end{array}\right)$ the family of all $i$-element subsets of $[n]$. The family $\cup_{i=0}^{s}\left(\begin{array}{c}{[n]} \\ i\end{array}\right)$ is $\left(\sum_{i=0}^{s-1}\left(\begin{array}{c}n-1 \\ i\end{array}\right)\right)$-regular. Thus we can include all of $\cup_{i=0}^{s}\left(\begin{array}{c}{[n]} \\ i\end{array}\right)$ in $\mathscr{F}$ and could still have some "available degree"; i.e. $d=k-1-\sum_{i=0}^{s-1}\left(\begin{array}{c}n-1 \\ i\end{array}\right)$ at each vertex.

We add to $\mathscr{F}$ several more sets, of size $s+1$, while making sure that the degree of no point grows above $k-1$, i.e. no point is contained in more than $d$ of these newly included $(s+1)$-element sets. To achieve this goal, we use a special case of the celebrated theorem of Baranyai [3], due to Katona.

Lemma 4.2. [4, Lemma, p. 179] Let $a \leq\left(\begin{array}{l}n \\ r\end{array}\right)$ be an arbitrary nonnegative integer. Then there exists an almost regular subhypergraph $\mathscr{H} \subseteq \mathscr{H}_{n}^{r}$ (that is the degrees of any two vertices in $\mathscr{H}$ differ by at most one) with $|E(\mathscr{H})|=$ a edges.

By choosing $r=s+1, a=\left\lfloor d \frac{n}{s+1}\right\rfloor$, Lemma 4.2 provides us with an almost regular $(s+1)$-uniform hypergraph $\mathscr{H}$ with $a$ edges. 


$$
\sum_{v \in V} \operatorname{deg}_{\mathscr{H}}(v)=D(\mathscr{H})=a(s+1) \leq d n
$$

As $\mathscr{H}$ is almost regular, no vertex has degree larger than $d$ in $\mathscr{H}$. Therefore $\mathscr{F}=\left(\cup_{i=0}^{s}\left(\begin{array}{c}{[n]} \\ i\end{array}\right)\right) \cup \mathscr{H}$ is a $k$-wise 0 -Fischer family with the required number of edges.

Let us assume now that there exists a $k$-wise 0 -Fischer family $\mathscr{F}^{\prime}$ with $\left|\mathscr{F}^{\prime}\right|=|\mathscr{F}|+1$. As $\mathscr{F}$ contains the first $|\mathscr{F}|$ smallest subsets of $[n], \sum_{A \in \mathscr{F}^{\prime}}|A|$ is larger than $\sum_{A \in \mathscr{F}}|A|$ by at least $\max \left\{|A|: A \in \mathscr{F}^{\prime}\right\} \geq s+1$,

$$
D\left(\mathscr{F}^{\prime}\right) \geq D(\mathscr{F})+s+1 .
$$

By our observation $D\left(\mathscr{F}^{\prime}\right) \leq(k-1) n$, giving us $D(\mathscr{F}) \leq(k-1) n-(s+1)$.

On the other hand by the definition of $\mathscr{F}$,

$$
\begin{aligned}
D(\mathscr{F}) & =\left\lfloor d \frac{n}{s+1}\right\rfloor(s+1)+(k-1-d) n \\
& >\left(\frac{d n}{s+1}-1\right)(s+1)+(k-1-d) n=(k-1) n-(s+1),
\end{aligned}
$$

a contradiction.

Hence we proved that $\mathscr{F}$ is a $k$-wise 0 -Fischer family of maximum size.

The next theorem determines $m_{l}^{k}(n)$ for most of the triples $(n, k, l)$; in particular when $k$ is fixed and $n$ is large enough. It reveals the fact that extremal $k$-wise $l$-Fischer families are not too exciting, as they are usually obtained from extremal $k$-wise 0 -Fischer families by the addition of $l$ new points to each member. The proof is basically identical to the one in Füredi [7]; we include an adaptation to our formulation of the statement.

Theorem 4.3. Let $2^{n}+1 \geq k \geq 2, n \geq l \geq 1$ be positive integers. Then

$$
\begin{array}{ll}
m_{l}^{k}(n)=\max \left\{m_{0}^{k}(n-l), k-1\right\} & \text { if } k+l>n \\
m_{l}^{k}(n) \leq \max \left\{m_{0}^{k}(n-l), n\right\} & \text { if } k+l \leq n,
\end{array}
$$

Proof. Clearly $m_{l}^{k}(n) \geq m_{0}^{k}(n-l)$, since from any $k$-wise 0 -Fischer family on $n-l$ points one can construct a $k$-wise $l$-Fischer family on $n$ points just by adding the same $l$ new points to each member.

Consider a $k$-wise $l$-Fischer family $\mathscr{F}=\left\{A_{1}, \ldots, A_{m}\right\}$ of size $m=m_{l}^{k}(n)$.

Case 1. There are $k-1$ sets in $\mathscr{F}$ whose intersection $B$ is of size $l$.

Then every member of $\mathscr{F}$ must contain $B$. Thus the family $\mathscr{F} \backslash B:=\{A \backslash B: A \in \mathscr{F}\}$ is a $k$-wise 0 -Fischer family on $n-l$ points, so $|\mathscr{F}|=|\mathscr{F} \backslash B| \leq m_{0}^{k}(n-l)$. Hence $m=m_{0}^{k}(n-l)$.

Case 2. The intersection of any $k-1$ members of $\mathscr{F}$ contains at least $l+1$ points.

Assume first that $k+l>n$. The value of $m$ cannot be less than $k-1$, since any collection of $k-1$ sets is a $k$-wise 0 -Fischer family. If $m>k-1$ then there exists 
an $l$-element set $B$, which is the intersection of $k$ members $A_{1}, \ldots A_{k}$ of $\mathscr{F}$. There are at most $k-1$ points in $[n] \backslash B$, so there exists an index $j \leq k$, such that the intersection of the $k-1$ sets $A_{1}, \ldots, A_{j-1}, A_{j+1}, \ldots, A_{k}$ is also $B$. This contradicts the assumption of Case 2 and proves $m=k-1$.

Assume now that $k+l \leq n$. Then clearly $k-1<n-l+1=m_{0}^{2}(n-l)$ $\leq m_{0}^{k}(n-l) \leq m$. Let $A_{1}, \ldots, A_{k-2} \in \mathscr{F}$ be members such that the cardinality of $B=A_{1} \cap \ldots \cap A_{k-2}$ is as small as possible. If there were $i>j>k-2$ such that $A_{i} \cap B=A_{j} \cap B$, that would imply $l=\left|A_{1} \cap \ldots A_{k-2} \cap A_{i} \cap A_{j}\right|=\left|B \cap A_{i}\right|$, contradicting our assumption that there are no $k-1$ members of the family having $l$ element intersection. Thus $A_{i} \cap B$ determines $A_{i}$ uniquely. The family $\mathscr{F}_{B}=\left\{B_{i}: B_{i}=A_{i} \cap B, i>k-2\right\}$, defined on $|B|$ points, is pairwise $l$-Fischer. Therefore the pairwise Nonuniform Fischer Inequality implies that it could have at most $|B|$ members. Since $|\mathscr{F}|=m \geq k$, there exists sets $A_{k-1}, A_{k} \in \mathscr{F}$. Every one of the $n-l$ points in $[n]-\cap_{i=1}^{k} A_{i}$ is not contained in at least one of the $A_{j} \mathrm{~s}, 1 \leq j \leq k$. A simple averaging argument then shows that the smallest $(k-2)$-wise intersection $B$ has cardinality at most $l+\frac{2}{k}(n-l)=n-\frac{k-2}{k}(n-l)$. So

$$
|\mathscr{F}|=\left|\mathscr{F}_{B}\right|+\mid\left\{A_{1}, \ldots, A_{k-2} \mid \leq n-\frac{k-2}{k}(n-l)+(k-2) \leq n-(k-2)\left(\frac{k}{n-l}-1\right) \leq n\right.
$$

Now Theorems 1.3 and 1.4 are simple corollaries of Lemma 4.1 and Theorem 4.3. We only remark that $m_{0}^{k}(n)$ is monotone increasing in $n$ and even $m_{0}^{k}(n-1)$ dominates both $k-1$ and $n$ in their respective range of interest.

\section{Remarks and Open Questions}

- We believe that Construction $\mathrm{A}$ is optimal for every $k \geq 3$ provided $n$ is large enough. The first unknown case is that of the 4-wise oddtown families, where the size of the extremal family is between $3 n-12$ and $3 n-9$.

- When a specific $L$ is given, it looks plausible that one can improve the constant multiplier 1 of the logarithm in Proposition 1.5. It is not clear what the sharp bound in this problem is, but probably the bound varies with $L$.

In the special case $L=\{0\}$ one can generalize Construction A which provides a family with $(k-1)\left(n-p \log _{2}(k-1)\right)$ members, such that the cardinality of any member is not divisible by $p$ but the cardinality of the intersection of any $k$ members is. We believe that (as in the case $p=2$ ) this lower bound is tight. However, there are nontrivial obstacles in modifying the proof of Theorem 1.2 to show this.

- A generalization of the construction in Theorem 1.3 for arbitrary $s=|L|$ is the following. Let $L=\{0,1, \ldots, s-1\}$. Let $\mathscr{G}_{s, k}$ be a maximum packing of $(s+1)$ element sets, such that each $s$-element set is contained in at most $k-2$ members of $\mathscr{G}_{s, k}$. The trivial combinatorial upper bound on $\left|\mathscr{G}_{s, k}\right|$ is $(k-2)\left(\begin{array}{l}n \\ s\end{array}\right) /(s+1)$. For $s=2$ and $k=3$, the infinite family of Steiner triple systems match this 
upper bound. In general, the existence of such tactical configurations is not known, but asymptotically optimal families could easily be constructed for every $k$ and $s=o(n)$ by generalizing the construction of [9, Theorem 8.1]. Let

$$
\mathscr{G}_{\alpha}=\left\{C \in\left(\begin{array}{c}
{[n]} \\
s+1
\end{array}\right): \sum_{c \in C} c \equiv \alpha n(\bmod n)\right\},
$$

where $\alpha$ is an integer. Then for any $k-2$ distinct integers $0 \leq \alpha_{1}<\alpha_{2}<\ldots$ $<\alpha_{k-2}<n, \cup_{i=1}^{k-2} \mathscr{C}_{\alpha_{i}}$ is a family covering each $s$-set at most $(k-2)$-times. By averaging, there exists a choice of $\alpha_{i}$ s for which $\mathscr{G}=\mathscr{G}_{s, k}:=\cup_{i=1}^{k-2} \mathscr{C}_{\alpha_{i}}$ has at least $(k-2)\left(\begin{array}{c}n \\ s+1\end{array}\right) / n$ members. Thus for $s=o(n),|\mathscr{G}|=\left(\frac{k-2}{s+1}+o(1)\right)\left(\begin{array}{c}n \\ s\end{array}\right)$. Then in the family $\mathscr{F}=\cup_{i=1}^{s}\left(\begin{array}{c}{[n]} \\ i\end{array}\right) \cup \mathscr{G}$ every $s$-set is contained in at most $k-1$ members, i.e. all the $k$-wise intersections are in $L$. The size of $\mathscr{F}$ is $\left(\frac{s+k-1}{s+1}+o(1)\right)\left(\begin{array}{l}n \\ s\end{array}\right)$. It is not hard to prove with a method similar to the one in Theorem 1.3 that for $L=\{0, \ldots, s-1\}$ $\mathscr{F}$, indeed, is asymptotically optimal. We conjecture that it is actually optimal for any $L,|L|=s$.

- One question we know very little about is the $k$-wise variant of the uniform RayChaudhuri-Wilson Theorem. Suppose we are given a set $L$ of $s$ integers. What is the size of a maximum family $\mathscr{F}$ of $l$-element sets, such that the cardinality of the intersection of any $k$ members of $\mathscr{F}$ is in $L$. The upper bound is still the usual $(k-1)\left(\begin{array}{l}n \\ s\end{array}\right)$, while we don't know any construction having more than $(1+o(1))\left(\begin{array}{l}n \\ s\end{array}\right)$ elements.

Acknowledgments. We are grateful to N. Alon and B. Sudakov for helpful discussions.

Note added in proof. Our above conjecture about the optimality of the size $\left(\frac{s+k-1}{s+1}+o(1)\right)\left(\begin{array}{l}n \\ s\end{array}\right)$ of the $L$-intersecting family $\mathscr{F}$ was settled recently by Füredi and Sudakov [10].

\section{References}

1. Alon, N., Babai, L., Suzuki, H.: Multilinear polynomials and Frankl-Ray-ChaudhuriWilson type intersection theorems. J. Comb. Theory, Ser. A, 58, 165-180 (1991)

2. Babai, L., Frankl, P.: Linear Algebra Methods in Combinatorics (manuscript)

3. Baranyai, Zs.: On the factorization of the complete uniform hypergraph, Infinite and finite sets (Colloq., Keszthely, 1973; dedicated to P. Erdös on his 60th birthday), Amsterdam, North-Holland: Colloq. Math. Soc. Janos Bolyai, 10, Vol. I, 91-108 (1975)

4. Katona, G.O.H.: On separating systems of a finite set. J. Comb. Theory, 1, 174-194 (1966)

5. Berlekamp, E.R.: On subsets with intersections of even cardinality. Can. Math.Bull. 12, 363-366 (1969)

6. Frankl, P., Wilson, R. M.: Intersection theorems with geometric consequences. Combinatorica. 1, 357-368 (1981) 
7. Füredi, Z.: On a problem of Deza and Frankl. Ars Comb. 13, 221-222 (1982)

8. Füredi, Z.: On finite set-systems whose every intersection is a kernel of a star. Discrete Math. 47, 129-132 (1983)

9. Füredi, Z.: Matchings and covers in hypergraphs. Graphs Comb. 4, 115-206 (1988)

10. Füredi, Z., Sudakov, B.: Extremal set systems with restricted $k$-wise intersections. J. Comb. Theory, Ser. A 105, 143-159 (2004)

11. Graver, J.E.: Boolean designs and self-dual matroids. Linear. Algebra. Appl. 10, $111-128(1975)$

12. Grolmusz, V.: Set-systems with restricted multiple intersections. Electron. J. Comb. 9, R8 (2002)

13. Grolmusz, V., Sudakov, B.: On $k$-wise set-intersections and $k$-wise Hamming-distances. J. Comb. Theory, Ser. A 99, 180-190 (2002)

14. Majumdar, K.N.: On some theorems in combinatorics relating to incomplete block designs. Ann. Math. Stat. 24, 377-389 (1953)

15. Sós, V.T.: Remarks on the connection of graph theory, finite geometry and block designs. Theorie Combinatorie, Proc. Colloq., Rome, Vol. II, Rome: Accad. Naz. Lincei, Atti dei Convegni Lincei 17, 223-233 (1973)

16. Vu, V.H.: Extremal set systems with upper bounded odd intersections. Graphs Comb. 13, 197-208 (1997)

17. Vu, V.H.: Set systems with weakly restricted intersections. Combinatorica 19, 567-587 (1999)

Received: December 16, 2002

Final version received: February 14, 2005 